DOI: https://doi.org/10.24297/jam.v18i.8600

\title{
Absolute Velocity and Total Stellar Aberration(II)
}

\author{
Miloš Čojanović \\ independent scholar \\ Montreal, Canada \\ cojmilmo@gmail.com
}

\begin{abstract}
In this paper, we will show that in addition to measuring annual and diurnal stellar aberration it is also possible directly to measure the angle of secular aberration caused by the motion of the solar system relative to other stars. In the manuscript [1] we dealt with this problem and gave a short description of a special telescope. Using such a telescope we would be able to measure the exact position of the cosmic objects and thus eliminate errors that occur due to the stellar aberration. Assuming that the tube of the telescope is filled with some optical medium [2], we will show that this does not significantly affect the measurement of the stellar aberration angle, but also that these differences are still large enough to enable us to determine the velocity at which the solar system moves relative to the other stars.
\end{abstract}

Keywords: Stellar Aberration, Absolute Velocity

\section{Introduction}

Suppose we observe an arbitrarily chosen star, which we denote by $(Z)$. Starlight moves in straight line and will remain in the same direction regarding to the ecliptic plane. Photons enter in a perpendicular direction to the top plane of the telescope.

The starlight represents an inertial frame of reference marked by $(K)$ and the telescope represents a moving frame of reference that is marked by $(T)$. We are assuming that [1] :

$P_{1}$ - speed of light in vacuum $c$ is constant and equal in all inertial frames $(K)$

$P_{2}$ - there is a one common time for the all frames $(\mathrm{K})$ and the moving frame $(T)$

$P_{3}$ - frame $(T)$ is moving uniformly in a straight line regarding the frame $(\mathrm{K})$

Suppose we have a telescope whose tube is filled with some matter whose index of refraction is denoted by $n$. The center of the upper surface of the telescope is indicated by the point $S$, and the center of its lower surface is indicated by the point $S^{\prime}$ [Figure 1]. 


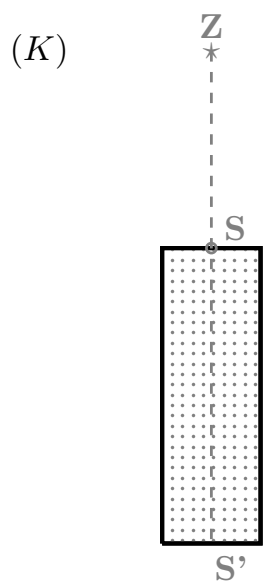

Figure 1: The telescope is stationary regarding the starlight

Let suppose the telescope moves relative to the starlight. At some instant $t_{0}$ photon hits the upper surface of the telescope at the point $\mathbf{S}$ and at some instant $t_{1}$, due to stellar aberration, it does not hit the lower surface of the telescope at point $\mathbf{S}^{\prime}$ but rather at the point $\mathbf{A}$ [Figure 2].

$(K)$

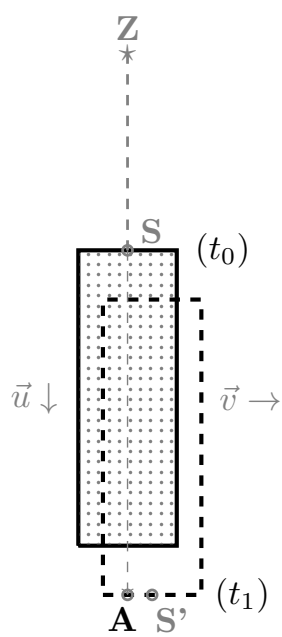

Figure 2: The telescope is moving regarding the starlight

Velocity at which the telescope moves relative to the starlight is decomposed to the two components. The first component noted by $\mathbf{v}$ is perpendicular to the starlight and the second one noted by $\mathbf{u}$ is parallel to starlight.

We will first find the distance that the photon has traveled in the direction of $\mathbf{u}$, regarding the $(\mathrm{K})$. Referring to the [Figure 2] we can write that: 


$$
\begin{array}{r}
d=S S^{\prime} \\
k=\frac{1}{n} \\
\Delta t=t_{1}-t_{0}
\end{array}
$$

$(k * c) * \Delta t=d+u * \Delta t \quad$ (the total distance traveled by a photon)

$$
\Delta t=\frac{d}{k * c-u}
$$

After that we are going to find the distance that the photon has traveled in the direction of $\mathbf{v}$, regarding the (K). In order to simplify the calculations, we assume that $\mathbf{u}=\mathbf{0}$. Let $S^{\prime}\left(t_{0}\right)$ denotes the position of the point $S^{\prime}$ in the instant $t_{0}$, and $S^{\prime}\left(t_{1}\right)$ denotes the position of the point $S^{\prime}$ in the instant $t_{1}$, regarding the $(\mathrm{K})$.

$S^{\prime}\left(t_{0}\right) S^{\prime}\left(t_{1}\right)=v * \Delta t \quad\left(\right.$ the total distance traveled by the point $\mathrm{S}^{\prime}$, regarding the $\left.(\mathrm{K})\right)$

$S^{\prime}\left(t_{0}\right) A=\frac{n-1}{n} * v * \Delta t \quad$ (the photon is dragged by the medium in the moving telescope - our hypothesis)

$$
\begin{array}{r}
S^{\prime} A=S^{\prime}\left(t_{1}\right) A=S^{\prime}\left(t_{1}\right) S^{\prime}\left(t_{0}\right)+S^{\prime}\left(t_{0}\right) A=-v * \Delta t+\frac{n-1}{n} * v * \Delta t= \\
=-\Delta t * \frac{v}{n}=-\frac{d *(v * k)}{k * c-u}=-\frac{v}{c-n * u} * d
\end{array}
$$

From the Equation (8) it follows that angle of the total stellar aberration [1] denoted by $\theta$ is equal to:

$$
\begin{array}{r}
\tan (\theta)=\left|\frac{S^{\prime} A}{d}\right|=\frac{d * v}{c-n * u} * \frac{1}{d}=\frac{v}{c-n * u} \\
\frac{v}{c-n * u}=\frac{v}{c} *\left(1+\frac{n u}{c}+\frac{n^{2} u^{2}}{c^{2}}+\ldots\right)=\frac{v}{c}+\frac{n u v}{c^{2}}+\frac{n^{2} u^{2} v}{c^{3}}+\ldots \\
\theta=\operatorname{atan} 2\left(\frac{v}{c-n * u}\right) \\
\theta \approx \frac{v}{c}+\frac{n u v}{c^{2}} \quad(\text { where } u<<c, v<<c)
\end{array}
$$

\section{Coordinate Systems}

In this section are given the descriptions of the four Coordinate Systems that will be used in a further discussion.

Let the $(P)$ represents "The Heliocentric-Ecliptic Coordinate System" [Figure 3]. Its origin $O_{p}$ is centered on the center of mass of the solar system, and the fundamental plane coincides with the ecliptic plane of the Earth's revolution about the sun. The line of intersection of the ecliptic plane and the earth's equatorial plane defines the $x_{p}-a x i s$. On the first day of Spring a line joining the center of the Earth and the center of the sun points in the direction of positive $x_{p}-$ axis [1].

Suppose that the point $O_{k}$ represents the position of the center of mass of the solar system (regarding the distant objects) at some point $T_{0}$ and by $O_{p}$ is marked the position of the center of mass of the solar system at the "present time" 
$T_{1}$. We will assume that the Coordinate Systems $(K)$ and $(P)$ are defined as "The Heliocentric-Ecliptic Coordinate System" except that their origins $O_{k}$ and $O_{p}$ are different. We assume that the Coordinate System $(P)$ is moving with a uniform, rectilinear space motion $\mathbf{u}$ relative to the Coordinate System $(K)$.

\section{$(P)$}

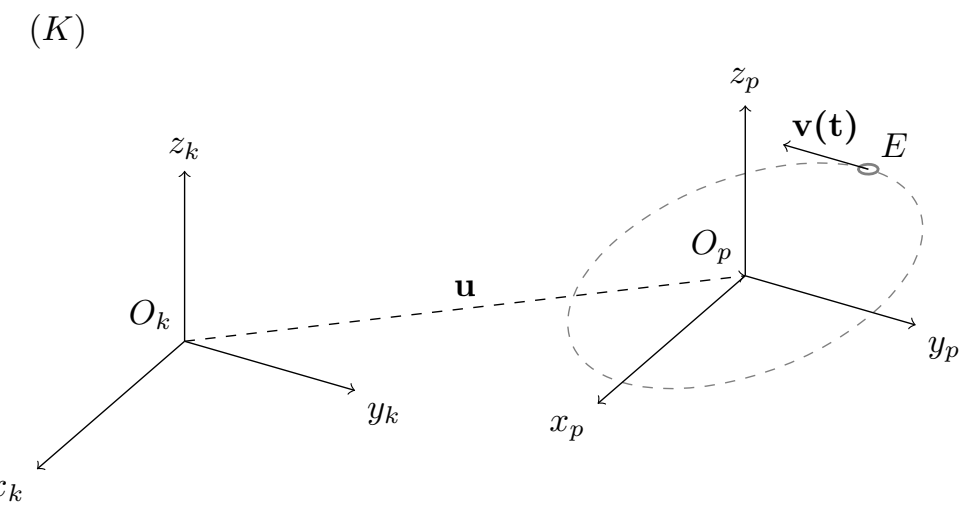

Figure 3: The Ecliptic Coordinate System $(P)$ moves uniformly regarding the Stationary Ecliptic Coordinate System $(K)$

Let a $(Q)$ represents "The Geocentric-Equatorial Coordinate System" Figure[4]. Its origin $O_{q}$ is at the center of the Earth, the fundamental plane is the equator and the positive $x_{q}$ points in the vernal equinox direction. The $z_{p}$ points in the direction of the north pole. By the definition the Coordinate System $(Q)$ is non-rotating with the respect to the stars [1].

Let $\varphi=23.43693 * \Pi / 180$ denotes Earth's axial tilt Figure[4]

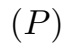

\section{$Q$}

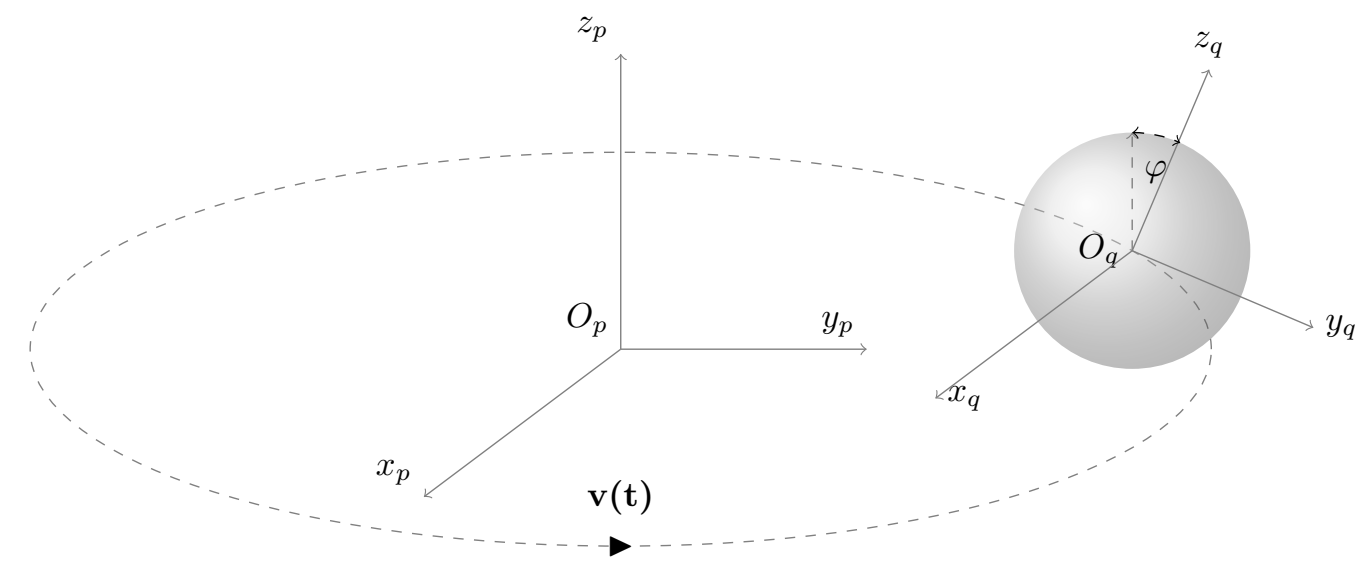

Figure 4: Ecliptic Coordinate System (P) and Equatorial Coordinate System (Q)

The position of the star is determined by two angles called right ascension and declination Figure[5]. The right ascension $\alpha$ is measured eastward in the plane of equator from the vernal equinox direction. The declination $\delta$ is 
measured northward from the equator to the line of sight, we would say that is an angle between the plane of equator and the direction of the starlight [1].

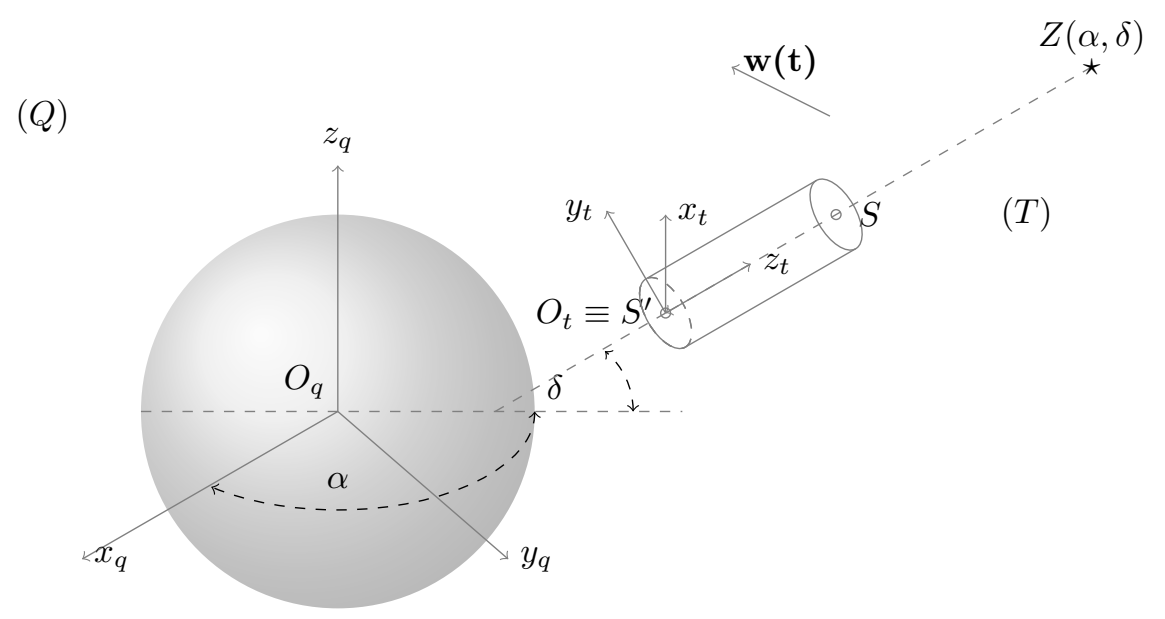

Figure 5: Equatorial Coordinate System (Q) and Telescope Coordinate System (T)

We will assume that the center line $S S^{\prime}$ of the telescope lies in the plane defined by the Earth's axis of rotation marked by $z_{q}$ and the star $Z(\alpha, \delta)$ Figure[5]. In addition, we will assume that $S S^{\prime}$ is parallel to the light rays coming from the star. We will define a right-handed Telescope Coordinate System $(T)$ system as follows. The origin of the Coordinate System $(T)$ is point $S^{\prime}$. The bottom plane of the telescope represents the $\left(x_{t} y_{t}\right)$ plane of the Coordinate System $(T)$. The positive $z_{t}$-axis lies in the $S^{\prime} S$ direction and the $x_{t}$-axis is parallel to the equatorial plane Figure[5].

\section{Coordinate Transformations}

In the previous paper [1], we have already derived transformation matrices that map Cartesian coordinates from one Coordinate System to another, but we will do it again in a different way.

We will first find simple formulas for transforming the arbitrarily chosen unit vector $\mathbf{a}=\mathbf{a}(\alpha, \delta)$ from a spherical to a Cartesian Coordinate System.

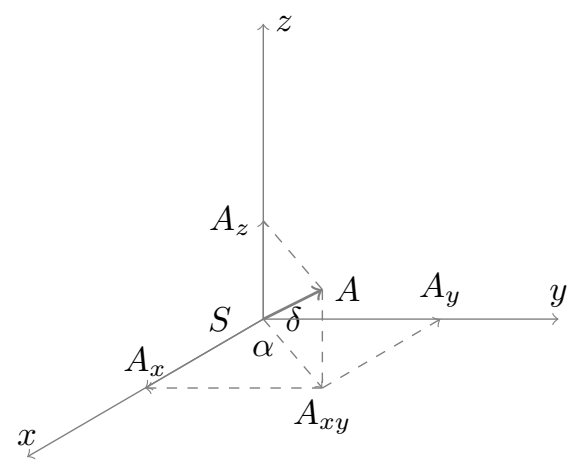

Figure 6: Transformation from Spherical to Cartesian coordinates 
As can be seen from the Figure[6] we have

$$
\begin{array}{r}
\mathbf{a}=\mathbf{a}(\alpha, \delta)=\left[a_{x}, a_{y}, a_{z}\right] \\
a_{x}=\cos (\alpha) * \cos (\delta) \\
a_{y}=\sin (\alpha) * \cos (\delta) \\
a_{z}=\sin (\delta)
\end{array}
$$

Where $\delta$ measures elevation from the $x y-$ plane.

Referring to the Figure[6] we have the following definitions and equations

$$
\begin{aligned}
& \theta_{x}=\angle A_{x} S A \\
& \theta_{x}=\angle A_{y} S A \\
& \theta_{z}=\angle A_{z} S A
\end{aligned}
$$

$$
\begin{array}{r}
\delta=\frac{\pi}{2}-\theta_{z} \\
\cos \left(\theta_{x}\right)=a_{x}=\cos (\alpha) * \cos (\delta) \\
\cos \left(\theta_{y}\right)=a_{y}=\sin (\alpha) * \cos (\delta) \\
\tan (\alpha)=\frac{a_{y}}{a_{x}}=\frac{\cos \left(\theta_{y}\right)}{\cos \left(\theta_{x}\right)} \\
\alpha=\operatorname{atan} 2\left(\frac{\cos \left(\theta_{y}\right)}{\cos \left(\theta_{x}\right)}\right)
\end{array}
$$

Let the triplets $\left[\hat{\mathbf{i}}_{k}, \hat{\mathbf{j}}_{k}, \hat{\mathbf{k}}_{k}\right] \mathrm{r}\left[\hat{\mathbf{i}}_{p}, \hat{\mathbf{j}}_{p}, \hat{\mathbf{k}}_{p}\right],\left[\hat{\mathbf{i}}_{q}, \hat{\mathbf{j}}_{q}, \hat{\mathbf{k}}_{q}\right]$ and $\left[\hat{\mathbf{i}}_{t}, \hat{\mathbf{j}}_{t}, \hat{\mathbf{k}}_{t}\right]$ represent the orthonormal bases for the Coordinate Systems $(K),(P),(Q)$ and $(T)$, respectively.

It is obvious that the unit matrix $I_{3}$ is transformation matrix from the basis $\left[\hat{\mathbf{i}}_{k}, \hat{\mathbf{j}}_{k}, \hat{\mathbf{k}}_{k}\right]$ to the basis $\left[\hat{\mathbf{i}}_{p}, \hat{\mathbf{j}}_{p}, \hat{\mathbf{k}}_{p}\right]$, which means that we have following equality:

$$
\left[\hat{\mathbf{i}}_{k}, \hat{\mathbf{j}}_{k}, \hat{\mathbf{k}}_{k}\right]=\left[\hat{\mathbf{i}}_{p}, \hat{\mathbf{j}}_{p}, \hat{\mathbf{k}}_{p}\right]
$$

Let the unit vectors $\hat{\mathbf{i}}_{q}, \hat{\mathbf{j}}_{q}$ and $\hat{\mathbf{k}}_{q}$ are expressed in spherical coordinates regarding the Ecliptic Coordinate System (P). Now using the Equations (13)-(16) we can transform them to the Cartesian Coordinates regarding the orthonormal basis $\left[\hat{\mathbf{i}}_{p}, \hat{\mathbf{j}}_{p}, \hat{\mathbf{k}}_{p}\right]$.

Referring to the Figure[4] we have: 


$$
\begin{array}{r}
\hat{\mathbf{i}}_{q}=\hat{\mathbf{i}}_{q}(0,0)=[\cos (0) * \cos (0), \sin (0) * \cos (0), \sin (0)]=[1,0,0] \\
\hat{\mathbf{j}}_{q}=\hat{\mathbf{j}}_{q}\left(\frac{\Pi}{2},-\varphi\right)=\left[\cos \left(\frac{\Pi}{2}\right) * \cos (-\varphi), \sin \left(\frac{\Pi}{2}\right) * \cos (-\varphi), \sin (-\varphi)\right]=[0, \cos (\varphi),-\sin (\varphi)] \\
\hat{\mathbf{k}}_{q}=\hat{\mathbf{k}}_{q}\left(\frac{\Pi}{2}, \frac{\Pi}{2}-\varphi\right)=\left[\cos \left(\frac{\Pi}{2}\right) * \cos \left(\frac{\Pi}{2}-\varphi\right), \sin \left(\frac{\Pi}{2}\right) * \cos \left(\frac{\Pi}{2}-\varphi\right), \sin \left(\frac{\Pi}{2}-\varphi\right)\right]= \\
=[0, \sin (\varphi), \cos (\varphi)]
\end{array}
$$

In that way we can define the matrix noted by $\kappa(Q, P)$ that transforms the basis $\left.\left[\hat{\mathbf{i}}_{p}, \hat{\mathbf{j}}_{p}, \hat{\mathbf{k}}_{p}\right]\right)$ to the basis $\left[\hat{\mathbf{i}}_{q}, \hat{\mathbf{j}}_{q}, \hat{\mathbf{k}}_{q}\right]$.

$$
\begin{gathered}
\kappa(Q, P)=\left[\begin{array}{ccc}
1 & 0 & 0 \\
0 & \cos (\varphi) & -\sin (\varphi) \\
0 & \sin (\varphi) & \cos (\varphi)
\end{array}\right] \\
{\left[\begin{array}{c}
\hat{\mathbf{i}}_{q} \\
\hat{\mathbf{j}}_{q} \\
\hat{\mathbf{k}}_{q}
\end{array}\right]=\kappa(Q, P) *\left[\begin{array}{c}
\hat{\mathbf{i}}_{p} \\
\hat{\mathbf{j}}_{p} \\
\hat{\mathbf{k}}_{p}
\end{array}\right]}
\end{gathered}
$$

If the $[\hat{\mathbf{i}}, \hat{\mathbf{j}}, \hat{\mathbf{k}}]$ represents orthonormal basis, then it is obvious that we have the following equalities:

$$
\begin{aligned}
& \hat{\mathbf{k}}=\hat{\mathbf{i}} \times \hat{\mathbf{j}} \\
& \hat{\mathbf{i}}=\hat{\mathbf{j}} \times \hat{\mathbf{k}} \\
& \hat{\mathbf{j}}=\hat{\mathbf{k}} \times \hat{\mathbf{i}}
\end{aligned}
$$

In other words, if two vectors are known then the third can be expressed as a cross product of the other two known vectors.

Let a matrix noted by $\kappa$ transforms an orthonormal basis $\left[\hat{\mathbf{i}}_{1}, \hat{\mathbf{j}}_{1}, \hat{\mathbf{k}}_{1}\right]$ to an orthonormal basis basis $\left[\hat{\mathbf{i}}_{2}, \hat{\mathbf{j}}_{2}, \hat{\mathbf{k}}_{2}\right]$. Then it is easy to prove the following equation [1].

$$
\kappa^{-1}=\kappa^{T}
$$

where $\kappa^{T}$ is transpose of matrix $\kappa$ 
Now we can find the matrix noted by $\kappa(P, Q)$ that transforms the basis $\left[\hat{\mathbf{i}}_{q}, \hat{\mathbf{j}}_{q}, \hat{\mathbf{k}}_{q}\right]$ to the basis $\left[\hat{\mathbf{i}}_{p}, \hat{\mathbf{j}}_{p}, \hat{\mathbf{k}}_{p}\right]$.

$$
\begin{gathered}
\kappa(P, Q)=\kappa^{-1}(Q, P)=\kappa^{T}(Q, P) \\
{\left[\begin{array}{c}
\hat{\mathbf{i}}_{p} \\
\hat{\mathbf{j}}_{p} \\
\hat{\mathbf{k}}_{p}
\end{array}\right]=\kappa^{T}(Q, P) *\left[\begin{array}{c}
\hat{\mathbf{i}}_{q} \\
\hat{\mathbf{j}}_{q} \\
\hat{\mathbf{k}}_{q}
\end{array}\right]}
\end{gathered}
$$

After that we are going to determine a transformation matrix noted by $\kappa(T, Q)$ that maps basis $\left[\hat{\mathbf{i}}_{q}, \hat{\mathbf{j}}_{q}, \hat{\mathbf{k}}_{q}\right]$ to the basis $\left[\hat{\mathbf{i}}_{t}, \hat{\mathbf{j}}_{t}, \hat{\mathbf{k}}_{t}\right]$.

Let the unit vectors $\hat{\mathbf{i}}_{t}, \hat{\mathbf{j}}_{t}$ and $\hat{\mathbf{k}}_{t}$ be expressed in spherical coordinates regarding the Equatorial Coordinate System (Q).

Referring to the Figure[5] it follows

$$
\begin{gathered}
\hat{\mathbf{i}}_{t}=\hat{\mathbf{i}}_{t}\left(\alpha+\frac{\Pi}{2}, 0\right)=\left[\cos \left(\alpha+\frac{\Pi}{2}\right) * \cos (0), \sin \left(\alpha+\frac{\Pi}{2}\right) * \cos (0), \sin (0)\right]=[-\sin (\alpha), \cos (\alpha), 0] \\
\hat{\mathbf{j}}_{t}=\hat{\mathbf{j}}_{t}\left(\alpha, \frac{\Pi}{2}+\delta\right)=\left[\cos (\alpha) * \cos \left(\frac{\Pi}{2}+\delta\right), \sin (\alpha) * \cos \left(\frac{\Pi}{2}+\delta\right), \sin \left(\frac{\Pi}{2}+\delta\right)\right] \\
=[-\cos (\alpha) * \sin (\delta),-\sin (\alpha) * \sin (\delta), \cos (\delta)] \\
\hat{\mathbf{k}}_{t}=\hat{\mathbf{k}}_{t}(\alpha, \delta)=[\cos (\alpha) * \cos (\delta), \sin (\alpha) * \cos (\delta), \sin (\delta)]
\end{gathered}
$$

In that way we can define the matrix noted by $\kappa(T, Q)$ that transforms $\left[\hat{\mathbf{i}}_{q}, \hat{\mathbf{j}}_{q}, \hat{\mathbf{k}}_{q}\right]$ to the basis $\left[\hat{\mathbf{i}}_{t}, \hat{\mathbf{j}}_{t}, \hat{\mathbf{k}}_{t}\right]$.

$$
\begin{gathered}
\kappa(T, Q)=\left[\begin{array}{ccc}
-\sin (\alpha) & \cos (\alpha) & 0 \\
-\cos (\alpha) * \sin (\delta) & -\sin (\alpha) * \sin (\delta) & \cos (\delta) \\
\cos (\alpha) * \cos (\delta) & \sin (\alpha) * \cos (\delta) & \sin (\delta)
\end{array}\right] \\
{\left[\begin{array}{c}
\hat{\mathbf{i}}_{t} \\
\hat{\mathbf{j}}_{t} \\
\hat{\mathbf{k}}_{t}
\end{array}\right]=\kappa(T, Q) *\left[\begin{array}{c}
\hat{\mathbf{i}}_{q} \\
\hat{\mathbf{j}}_{q} \\
\hat{\mathbf{k}}_{q}
\end{array}\right]}
\end{gathered}
$$


Now we can define the matrix noted by $\kappa(Q, T)$ that transforms $\left[\hat{\mathbf{i}}_{t}, \hat{\mathbf{j}}_{t}, \hat{\mathbf{k}}_{t}\right]$ to the basis $\left[\hat{\mathbf{i}}_{q}, \hat{\mathbf{j}}_{q}, \hat{\mathbf{k}}_{q}\right]$.

$$
\begin{gathered}
\kappa(Q, T)=\kappa^{-1}(T, Q)=\kappa^{T}(T, Q) \\
{\left[\begin{array}{c}
\hat{\mathbf{i}}_{q} \\
\hat{\mathbf{j}}_{q} \\
\hat{\mathbf{k}}_{q}
\end{array}\right]=\kappa^{T}(T, Q) *\left[\begin{array}{c}
\hat{\mathbf{i}}_{t} \\
\hat{\mathbf{j}}_{t} \\
\hat{\mathbf{k}}_{t}
\end{array}\right]}
\end{gathered}
$$

It is easy to prove following equation:

$$
\left[\begin{array}{c}
\hat{\mathbf{i}}_{t} \\
\hat{\mathbf{j}}_{t} \\
\hat{\mathbf{k}}_{t}
\end{array}\right]=\kappa(T, Q) * \kappa(Q, P) *\left[\begin{array}{c}
\hat{\mathbf{i}}_{p} \\
\hat{\mathbf{j}}_{p} \\
\hat{\mathbf{k}}_{p}
\end{array}\right]
$$

Now we can determine the matrix noted by $\kappa(T, P)$ that transforms the basis $\left[\hat{\mathbf{i}}_{p}, \hat{\mathbf{j}}_{p}, \hat{\mathbf{k}}_{p}\right]$ to the basis $\left[\hat{\mathbf{i}}_{t}, \hat{\mathbf{j}}_{t}, \hat{\mathbf{k}}_{t}\right]$

$$
\kappa(T, P)=\kappa(T, Q) * \kappa(Q, P)
$$

and the matrix noted by $\kappa(P, T)$ that transforms $\left[\hat{\mathbf{i}}_{t}, \hat{\mathbf{j}}_{t}, \hat{\mathbf{k}}_{t}\right]$ to the $\left[\hat{\mathbf{i}}_{p}, \hat{\mathbf{j}}_{p}, \hat{\mathbf{k}}_{p}\right]$.

$$
\begin{gathered}
\kappa(P, T)=\kappa^{-1}(T, P)=\kappa^{T}(Q, P) * \kappa^{T}(T, Q) \\
{\left[\begin{array}{c}
\hat{\mathbf{i}}_{p} \\
\hat{\mathbf{j}}_{p} \\
\hat{\mathbf{k}}_{p}
\end{array}\right]=\kappa^{T}(Q, P) * \kappa^{T}(T, Q) *\left[\begin{array}{c}
\hat{\mathbf{i}}_{t} \\
\hat{\mathbf{j}}_{t} \\
\hat{\mathbf{k}}_{t}
\end{array}\right]}
\end{gathered}
$$

Let a matrix noted by $\kappa$ transforms an orthonormal basis $\left[\hat{\mathbf{i}}_{1}, \hat{\mathbf{j}}_{1}, \hat{\mathbf{k}}_{1}\right]$ to an orthonormal basis $\left[\hat{\mathbf{i}}_{2}, \hat{\mathbf{j}}_{2}, \hat{\mathbf{k}}_{2}\right]$ and suppose that we have some arbitrary vector denoted by $\mathbf{v}$. Then we have the following equations :

$$
\left[\begin{array}{l}
\hat{\mathbf{i}}_{2} \\
\hat{\mathbf{j}}_{2} \\
\hat{\mathbf{k}}_{2}
\end{array}\right]=\kappa *\left[\begin{array}{l}
\hat{\mathbf{i}}_{1} \\
\hat{\mathbf{j}}_{1} \\
\hat{\mathbf{k}}_{1}
\end{array}\right]
$$




$$
\mathbf{v}=v_{x} * \hat{\mathbf{i}}_{1}+v_{y} * \hat{\mathbf{j}}_{1}+v_{z} * \hat{\mathbf{z}}_{1}=\left[\begin{array}{lll}
v_{x} & v_{y} & v_{z}
\end{array}\right] *\left[\begin{array}{l}
\hat{\mathbf{i}}_{1} \\
\hat{\mathbf{j}}_{1} \\
\hat{\mathbf{k}}_{1}
\end{array}\right]=\left[\begin{array}{lll}
v_{x} & v_{y} & v_{z}
\end{array}\right] * \kappa^{T} *\left[\begin{array}{c}
\hat{\mathbf{i}}_{2} \\
\hat{\mathbf{j}}_{2} \\
\hat{\mathbf{k}}_{2}
\end{array}\right]
$$

Using the Equation (49), it is easy to map the vector $\mathbf{v}$ from one basis to another.

\section{Determining the actual position of the star and its total stellar aber- ration}

Suppose there is a telescope (satellite) moving at a velocity $\mathbf{w}(t)$ with respect to the Equatorial Coordinate System (Q), Figure[5] and Figure[7].

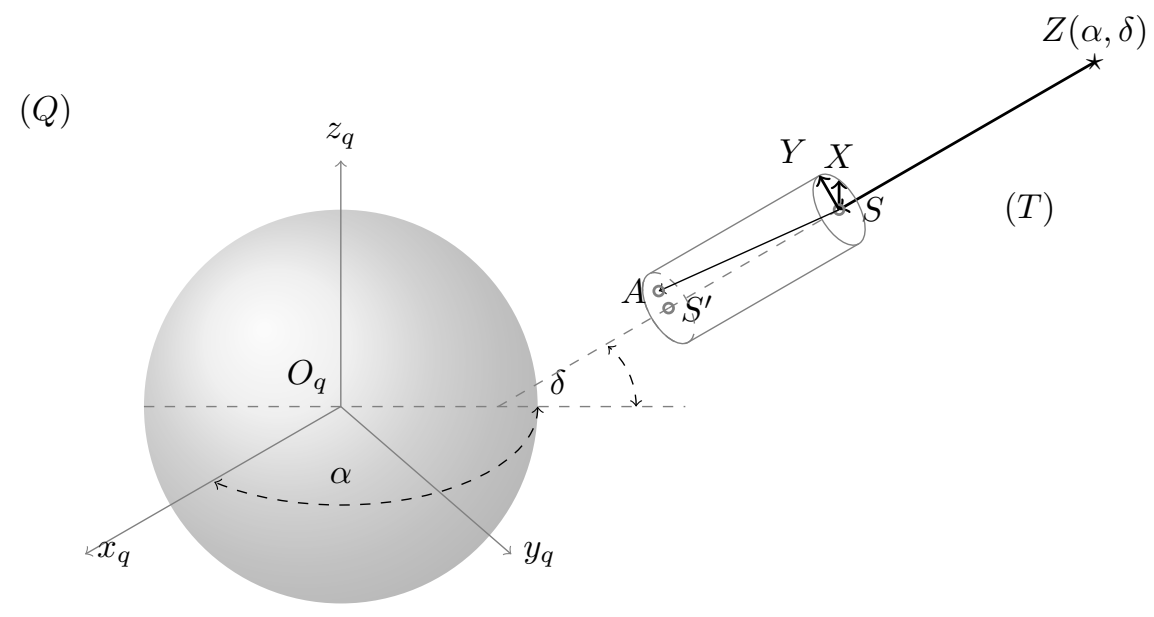

Figure 7: Determining the actual right ascension $\alpha$, declination $\delta$ and total aberration of the star

We will assume that at point $S$ there is a splitter, so that the light ray emitted from star $Z$ is sent simultaneously in two directions $S X$ and $S Y$ where $(S X=S Y$ and $S X \perp S Y)$, Figure[7]. The rays $S X$ and $S Y$ are perpendicular to $S S^{\prime}$ which implies that the points $Z, S$ and $S^{\prime}$ lie on one line. Now we can measure the angles $\theta_{x}, \theta_{y}$ and $\theta_{z}$ between $S^{\prime} S$ and the positive $x_{q}, y_{q}$ and $z_{q}$ axes, respectively. Applying the Equations (23) - (24) it follows that :

$$
\begin{array}{r}
\delta=\frac{\Pi}{2}-\theta_{z} \\
\alpha=\operatorname{atan} 2\left(\frac{\cos \left(\theta_{y}\right)}{\cos \left(\theta_{x}\right)}\right)
\end{array}
$$

The angles $\alpha$ and $\delta$ are known and using the Equations $(37)-(39)$ we are able to define a basis $\left[\hat{\mathbf{i}}_{t}, \hat{\mathbf{j}}_{t}, \hat{\mathbf{k}}_{t}\right]$ regarding the Telescope Coordinate System (T).

Let the telescope remains in the same position, which means that points $Z, S$ and $S^{\prime}$ still lie on the same line. Instead of sending beam to points $X, Y$ we will let the light signal continue moving toward bottom surface of the telescope. 
Due to stellar aberration instead of point $S^{\prime}$, the light signal will hit point $A$.

Now, knowing the vector $\mathbf{S}^{\prime} \mathbf{A}$ our goal is to determine a velocity at which the telescope moves relative to Coordinate System (K).

\section{Determining a velocity at which the telescope moves relative to Co- ordinate System $(\mathbf{K})$}

The velocity at which the telescope moves with respect to Coordinate System $(\mathrm{K})$ will be denoted by $\mathbf{U}(t)$. We have the following equations:

$$
\begin{array}{r}
\mathbf{U}(t)=\left[U_{x}(t), U_{y}(t), U_{z}(t)\right] \\
\mathbf{U}(t)=\mathbf{u}+\mathbf{v}(t)+\mathbf{w}(t)
\end{array}
$$

Refer to Figure[3] it follows that the Coordinate System $(P)$ moves at a uniform velocity $\mathbf{u}$ relative to the Coordinate System $(K)$, which will be noted in the following way.

$$
\mathbf{u}=\left[u_{x}, u_{y}, u_{z}\right]
$$

Where $u_{x}, u_{y}, u_{z}$ are the coordinates of $\mathbf{u}$ over $\left[\hat{\mathbf{i}}_{k}, \hat{\mathbf{j}}_{k}, \hat{\mathbf{k}}_{k}\right]$. Now we are going to transform the coordinates $u_{x}, u_{y}, u_{z}$ of the vector $\mathbf{u}$ with respect to the basis $\left[\hat{\mathbf{i}}_{k}, \hat{\mathbf{j}}_{k}, \hat{\mathbf{k}}_{k}\right]$ to the coordinates $u_{x}[T], u_{y}[T], u_{z}[T]$ with respect to the basis $\left[\hat{\mathbf{i}}_{t}\right.$, $\left.\hat{\mathbf{j}}_{t}, \hat{\mathbf{k}}_{t}\right]$.

$$
\mathbf{u}=\left[u_{x}[T], u_{y}[T], u_{z}[T]\right]=\left[u_{x}, u_{y}, u_{z}\right] * \kappa^{T}(Q, P) * \kappa^{T}(T, Q)
$$

Refer to Figure[4] it follows that the Coordinate System $(Q)$ moves at velocity $\mathbf{v}(t)$ relative to the Coordinate System $(P)$, which will be noted in the following way.

$$
\mathbf{v}(t)=\left[v_{x}, v_{y}, v_{z}\right]
$$

Now we are going to transform the coordinates $v_{x}, v_{y}, v_{z}$ of the vector $\mathbf{v}(t)$ with respect to the basis $\left[\hat{\mathbf{i}}_{p}, \hat{\mathbf{j}}_{p}, \hat{\mathbf{k}}_{p}\right]$ to the coordinates $v_{x}[T], v_{y}[T], u_{v}[T]$ with respect to the basis $\left[\hat{\mathbf{i}}_{t}, \hat{\mathbf{j}}_{t}, \hat{\mathbf{k}}_{t}\right]$.

$$
\mathbf{v}(t)=\left[v_{x}[T], v_{y}[T], v_{z}[T]\right]=\left[v_{x}, v_{y}, v_{z}\right] * \kappa^{T}(Q, P) * \kappa^{T}(T, Q)
$$


As can be seen from the Figure[5], the Coordinate System $(T)$ moves at velocity $\mathbf{w}(t)$ relative to the Coordinate System $(Q)$, which will be noted in the following way.

$$
\mathbf{w}(t)=\left[w_{x}, w_{y}, w_{z}\right]
$$

After that we are going to transform the coordinates $w_{x}, w_{y}, w_{z}$ of the vector $\mathbf{w}(t)$ with respect to the basis $\left[\hat{\mathbf{i}}_{q}, \hat{\mathbf{j}}_{q}, \hat{\mathbf{k}}_{q}\right]$ to the coordinates $w_{x}[T], w_{y}[T], w_{z}[T]$ with respect to the basis $\left[\hat{\mathbf{i}}_{t}, \hat{\mathbf{j}}_{t}, \hat{\mathbf{k}}_{t}\right]$.

$$
\mathbf{w}(t)=\left[w_{x}[T], w_{y}[T], w_{z}[T]\right]=\left[w_{x}, w_{y}, w_{z}\right] * \kappa^{T}(T, Q)
$$

And finally, by applying the Equations (55),(57) and (59) we are able to express the coordinates $U_{x}, U_{y}, U_{z}$ of the vector $\mathbf{U}(t)$ with respect to the basis $\left[\hat{\mathbf{i}}_{k}, \hat{\mathbf{j}}_{k}, \hat{\mathbf{k}}_{k}\right]$ to the coordinates noted by $U_{x}[T], U_{y}[T], U_{z}[T]$ with respect to the basis $\left[\hat{\mathbf{i}}_{t}, \hat{\mathbf{j}}_{t}, \hat{\mathbf{k}}_{t}\right]$.

$$
\begin{array}{r}
U_{x}[T]=u_{x}[T]+v_{x}[T]+w_{x}[T] \\
U_{y}[T]=u_{y}[T]+v_{y}[T]+w_{y}[T] \\
U_{z}[T]=u_{z}[T]+v_{z}[T]+w_{z}[T] \\
U[T]=\left[U_{x}[T], U_{y}[T], U_{z}[T]\right]
\end{array}
$$

In order to find the vector $\mathbf{u}$ we first need to determine the vector $\mathbf{U}[\mathrm{T}]$.

\section{Determining a velocity that solar system moves regarding the Coor- dinate System (K)}

We will first define the Coordinate System $\left(K^{\prime}\right)$ whose origin is at the point $O_{k}$ and the axes $x_{k}^{\prime}, y_{k}^{\prime}, z_{k}^{\prime}$ determined by the basis $\left[\hat{\mathbf{i}}_{t}, \hat{\mathbf{j}}_{t}, \hat{\mathbf{k}}_{t}\right]$. In other words, the center of the Coordinate System $\left(K^{\prime}\right)$ coincides with the center $O_{k}$ of the Coordinate System $(K)$, and the coordinate axes parallel to the coordinate axes of the Coordinate System $(T)$.

Referring to the Figure[7 it follows that on the bottom side of the telescope we will have the situation shown in the Figure[8]. 


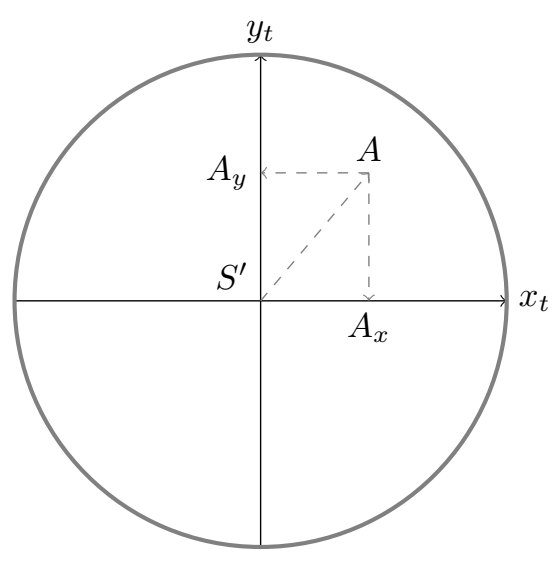

Figure 8: Due to the stellar aberration photons hit bottom side of the telescope at point $A$

The origin of the Telescope Coordinate System $(T)$ is noted by $S^{\prime}$ Figure[8], its axes are marked by $x_{t}$ and $y_{t}, A$ denotes the point where light hits the bottom plane of the telescope, points $A_{x}$ and $A_{y}$ are the projections of point $A$ on the $x_{t}$ and $y_{t}$ axes.

Suppose we have two telescopes filled with two different materials whose indices of refraction are equal to $n_{0}$ and $n_{1}$ respectively. We can actually say that we have one telescope with two tubes. The tube may be located in the primary or secondary position. When the tube is in the primary position then using that tube it is possible to make measurements.

If $\left|S^{\prime} A\right|=0$ we can conclude that the proposed method did not produce the expected results and therefore we can say that the experiment failed.

Otherwise from the Equation (8) and the Figure[8] we can find that:

$$
\begin{array}{r}
S^{\prime} A_{x}\left(n_{0}\right)=\frac{U_{x}[T]}{c-n_{0} * U_{z}[T]} * d \\
S^{\prime} A_{x}\left(n_{1}\right)=\frac{U_{x}[T]}{c-n_{1} * U_{z}[T]} * d \\
U_{z}[T]=c * \frac{S^{\prime} A_{x}\left(n_{0}\right)-S^{\prime} A_{x}\left(n_{1}\right)}{n_{0} * S^{\prime} A_{x}\left(n_{0}\right)-n_{1} * S^{\prime} A_{x}\left(n_{1}\right)} \\
U_{x}[T]=\frac{S^{\prime} A_{x}\left(n_{0}\right) *\left(c-n_{0} * U_{z}[T]\right)}{d} \\
S^{\prime} A_{y}\left(n_{0}\right)=\frac{U_{y}[T]}{c-n_{0} * U_{z}[T]} * d \\
S^{\prime} A_{y}\left(n_{1}\right)=\frac{U_{y}[T]}{c-n_{1} * U_{z}[T]} * d \\
U_{z}[T]=c * \frac{S^{\prime} A_{y}\left(n_{0}\right)-S^{\prime} A_{y}\left(n_{1}\right)}{n_{0} * S^{\prime} A_{y}\left(n_{0}\right)-n_{1} * S^{\prime} A_{y}\left(n_{1}\right)} \\
U_{y}[T]=\frac{S^{\prime} A_{y}\left(n_{0}\right) *\left(c-n_{0} * U_{z}[T]\right)}{d}
\end{array}
$$


If our measurements and calculations are correct then the following identity holds:

$$
\frac{S^{\prime} A_{x}\left(n_{0}\right)-S^{\prime} A_{x}\left(n_{1}\right)}{n_{0} * S^{\prime} A_{x}\left(n_{0}\right)-n_{1} * S^{\prime} A_{x}\left(n_{1}\right)}=\frac{S^{\prime} A_{y}\left(n_{0}\right)-S^{\prime} A_{y}\left(n_{1}\right)}{n_{0} * S^{\prime} A_{y}\left(n_{0}\right)-n_{1} * S^{\prime} A_{y}\left(n_{1}\right)}
$$

From the Equations (60) - (62) it follows that:

$$
\begin{aligned}
& u_{x}[T]=U_{x}[T]-v_{x}[T]-w_{x}[T] \\
& u_{y}[T]=U_{y}[T]-v_{y}[T]-w_{y}[T] \\
& u_{z}[T]=U_{z}[T]-v_{z}[T]-w_{z}[T]
\end{aligned}
$$

And from the Equation (55) we have:

$$
\mathbf{u}=\left[u_{x}, u_{y}, u_{z}\right]=\left[u_{x}[T], u_{y}[T], u_{z}[T]\right] * \kappa(T, Q) * \kappa(Q, P)
$$

In this way we have found the velocity $\mathbf{u}$ at which the Coordinate System $(P)$ moves with respect to Coordinate System $(K)$.

\section{Analysis of the results}

It remains to determine what velocity $\mathbf{u}$ actually represents.

Suppose we observed $n$ different stars from our Galaxy and performed all the necessary measurements and calculations. In that way we got a sequence of vectors:

$\left\{\mathbf{u}_{i}\right\}_{i=1}^{n}$

Let $u_{E}$ denotes some expected minimal value of the $\left\|\mathbf{u}_{i}\right\|$, and $\varepsilon$ denotes some small positive number.

Based on the obtained values of $\mathbf{u}_{i}$, we will consider three cases:

1. $\left\|\mathbf{u}_{i}\right\|<u_{E} \quad\{i=1,2, . . n\}$

This means that we have a following identity:

$$
\mathbf{u}=\mathbf{0}
$$

In this case, we are not able to detect any movement of the solar system regarding to other stars. Therefore, this movement has no effect on stellar aberration.

2. $\left\|\mathbf{u}_{i}-\mathbf{u}_{j}\right\| \leq \varepsilon \quad\{i, j=1,2, . . n\}$

In this case we will define a vector $\mathbf{u}$ as follows:

$$
\mathbf{u}=\frac{\sum_{i=1}^{n} \mathbf{u}_{i}}{n}
$$

Obviously, in this case we can say that velocity $\mathbf{u}$ has an absolute value and the motion of the solar system regarding to other stars affects stellar aberration. 
3. $\left\|\mathbf{u}_{i}-\mathbf{u}_{j}\right\|>\varepsilon \quad$ (for some $\left.i, j\right)$

In this case we can say that $\mathbf{u}_{i}$ represents the relative velocity at which the solar system moves with respect to the observed star and that this motion affects stellar aberration.

We can repeat the experiment by observing objects outside our Galaxy and make comparisons between the obtained results.

\section{References}

[1] Čojanović M.(2018) Absolute Velocity and Total Stellar Aberration Journal of Applied Mathematics and Physics, 6, 1034-1054. https://doi.org/10.4236/jamp.2018.65090

[2] Elio Antonello (2014) Water-filled telescopes https://arxiv.org/ftp/arxiv/papers/1401/1401.5585.pdf 